nature

immunology

\title{
Elements of a good decision
}

\author{
Signal integration, network complexities and genetic programming render the immune system ready to react to \\ challenges from without and within.
}

$T$

he complexity of decision making in the immune system is illustrated by the controversy surrounding the CD4 versus CD8 T cell lineage choice in the thymus. The long-lasting debate between a stochastic or selective model versus an instructive model has elegantly evolved into what we now call the kinetic signaling model of lineage choice, which proposes that asymmetric changes in $\mathrm{CD} 4$ and $\mathrm{CD} 8$ expression induced by signaling from the $\mathrm{T}$ cell antigen receptor cause distinct persistence of the signal depending on the class of MHC that engages the receptor. Signals mediated by the $\mathrm{T}$ cell receptor and those initiated by interleukin-7, a cytokine produced in a localized manner by the thymic stroma, are integrated and translated into antagonistic transcriptional circuitries that decide on one lineage or another. Three main conclusions can be drawn from this particular lineage checkpoint that can be applied to cell fate decisions in general: cells integrate a multitude of signals, with most inputs being combinatorial (in time or space), not linear; context matters, in that no cell is ever ignorant of its environment; and there is much more complexity than we ever imagined, and induction of a signal can require inhibition of an inhibitor rather than triggering of an activator or, most frequently, a combination of both.

Although there may still be much to be learned about the full complexity, integrative capacity and proactiveness of the immune system, substantial progress has been made in recent years in understanding how immune cells 'make a choice'. It is thus timely to introduce the current Focus on decision making in the immune system (http://www.nature. com/ni/focus/decisionmaking/), which summarizes the most recent concepts and latest advances in the field. The five reviews commissioned for this purpose discuss the integration of various environmental instructions and intrinsic programs that create a successful immune response. They cover various areas, from the role of innate immune system and nonlymphoid tissue in orchestrating the adaptive immune response, to plasticity of effector $\mathrm{T}$ cell subsets and checkpoints in antibody production by $\mathrm{B}$ cells. The microenvironmental conditions, signals, receptors, transcriptional networks and biological outcomes of these immune responses are comprehensively presented in the five reviews.

Bali Pulendran and colleagues discuss the fundamental role of the innate immune system in sensing pathogens and tuning the quality of helper $\mathrm{T}$ cell responses. In contrast to the significant progress made in understanding the role of innate immunity in inducing $\mathrm{T}_{\mathrm{H}} 1$ and $\mathrm{T}_{\mathrm{H}} 17$ responses, much less is known about its role in initiating $\mathrm{T}_{\mathrm{H}} 2$ and tolerogenic responses. The review summarizes the emerging insights into the role of dendritic cell subsets, pathogen recognition receptors, signaling pathways and accessory cell types that orchestrate $\mathrm{T}_{\mathrm{H}} 2$ and tolerogenic responses and offers a conceptual framework for understanding the innate control of helper $\mathrm{T}$ cell responses.

The view, held for many years, that the body surface epithelia contribute to host protection strictly as a physical and chemical barrier is being revised owing to accumulating evidence that epithelial cells can activate tissue-associated lymphocytes and that the epithelial cells' response to infection and/or stress can strongly influence dendritic cells and subsequent adaptive immune responses. Starting from these premises, Wendy Havran, Adrian Hayday and colleagues assess our current understanding of the key afferent stimuli, epithelial cell pathways and type of cell-bound or secreted signals that can communicate the state of the epithelium to the immune compartment and regulate immunity.

An entire review is dedicated to decision checkpoints in the complex environment of the thymus. Andrea Carpenter and Rémy Bosselut focus on the signals that direct $\alpha \beta$ and $\gamma \delta$ thymocyte divergence and the positive selection of $\alpha \beta$ thymocytes. They thoroughly discuss the sensors and transcriptional circuitries that can convert these signals into lineage decisions.

Finally, two reviews discuss aspects of decision making in mature $\mathrm{T}$ and $\mathrm{B}$ lymphocytes. As new functional states of helper T cells are being characterized, Brigitta Stockinger and Kenneth Murphy provide a framework for explaining the relative flexibility or stability of distinct $\mathrm{T}$ cell subsets. By constructing an analogy between CD4 subsets and the energy levels in an atom, the authors analyze the factors that contribute to subset stability or plasticity. They define four regulatory components (conditions, transcriptional circuitry, clonality and chromatin state) that can explain the differentiation, maintenance and plasticity of a phenotype. In the final review of the Focus, Christopher Goodnow and colleagues describe our current understanding of control systems and fail-safe mechanisms that guide antibody production by $\mathrm{B}$ cells. The choices between survival, death, differentiation and proliferation are discussed at key points during $B$ cell life-from the moment of antigen encounter to memory or plasma cell differentiation.

Cells in complex organisms continually face decisions about differentiation, proliferation, quiescence, stress responses and apoptosis. Decisions triggered by direct signals will always be influenced by indirect factors, such as environmental context, differentiation stage and metabolic state of the cell. Because of the dynamic and interactive nature of the immune system, immune cells constantly 'decide' on how to deal best with invading pathogens and other homeostatic challenges. The Focus summarizes our current view of the sensors, networks and key cellular players involved in immune decision making. Understanding the environmental, cellular and molecular mechanisms that control this process will be essential for the rational design of vaccines and therapeutics. 